Mean follow-up was 24.4 months. The MBDA-based CVD risk score (mean 3.3, IQR 2.8-3.8) was highly significant in univariate analysis, with $\mathrm{HR}=3.99(95 \%$ $\mathrm{Cl}: 3.52-4.51, \mathrm{p}=4.4 \times 10^{-95}$ ); i.e., for every 1 -unit increase in risk score, the CVD event rate in this cohort was $\sim 4$ times as high. Similar results were seen in the subset of 44,379 patients $<65$ years old, with $\mathrm{HR}=4.26$ (95\% Cl: $3.53-5.14$, $\left.p=1.2 \times 10^{-47}\right)$. In sensitivity analyses, after adjusting for multiple comparisons, there were no significant differences between $\mathrm{HR}$ of complementary subgroups (Figure 1). The MBDA-based CVD risk score added significant prognostic information to a simpler, clinical model $\left(\mathrm{HR}=2.28\left[95 \% \mathrm{Cl}: 1.69-3.08, \mathrm{p}=1.6 \times 10^{-7}\right]\right.$ after accounting for all other factors).

Conclusion: The MBDA-based CVD risk prediction score has been externally validated in a cohort that is younger than and independent of the Medicare cohort used previously for test development and internal validation.

Table 1. Cohort characteristics of RA patients with linked biomarker data and at risk for CVD events.

\begin{tabular}{lc}
\hline Variable & Median (IQR) or N (\%) \\
\hline Total patients & $48,868()$ \\
Age, years & $54(46-60)$ \\
Sex, male & $8,940(18.3 \%)$ \\
Diabetes & $7,974(16.3 \%)$ \\
Hypertension & $19,132(39.2 \%)$ \\
History of high-risk CVD event & $6,713(13.7 \%)$ \\
Smoking & $7,487(15.3 \%)$ \\
CRP, mg/L & $4.1(1.4-11.5)$ \\
Leptin, ng/mL & $24.3(10.6-47.1)$ \\
MMP-3, ng/mL & $21.1(14.3-36.2)$ \\
TNF-RI, ng/mL & $1.4(1.1-1.7)$ \\
MBDA score & $40(31-48)$ \\
MBDA-based CVD risk score & $3.3(2.8-3.8)$ \\
\hline
\end{tabular}

Figure 1. Hazard ratios for CVD event over 3 years in the external validation dataset $(\mathrm{N}=48,868)$. Vertical dashed line indicates the hazard ratio for all patients.

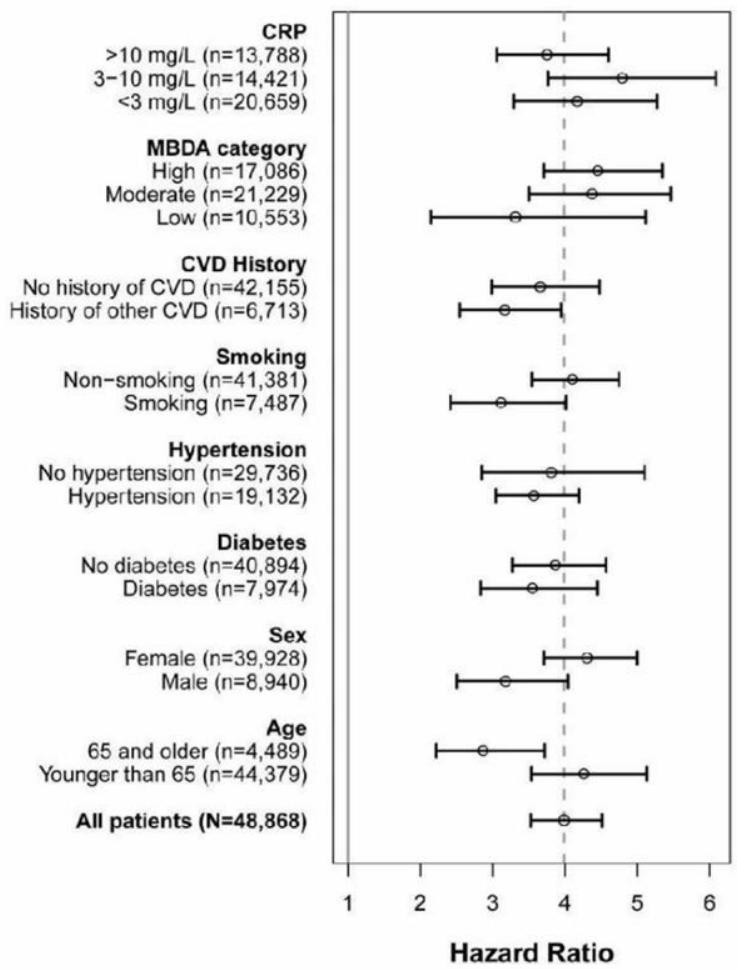

Disclosure of Interests: Jeffrey Curtis Grant/research support from: Abbvie, Amgen, BMS, Corrona, Eli Lilly, Jannsen, Myriad Genetics, Inc., Pfizer, Regeneron, Roche, and UCB., Eric Sasso Shareholder of: Myriad
Genetics, Inc., Employee of: Myriad Autoimmune, Elena Hitraya Shareholder of: Myriad Genetics, Inc., Employee of: Myriad Autoimmune, Cheryl Chin Shareholder of: Myriad Genetics, Inc., Employee of: Myriad Autoimmune Richard Bamford Shareholder of: Myriad Genetics, Inc., Employee of: Myriad Autoimmune, Rotem Ben-Shachar Shareholder of: Myriad Genetics, Inc. Employee of: Myriad Genetics, Inc., Alexander Gutin Shareholder of: Myriad Genetics, Inc., Employee of: Myriad Genetics, Inc., Darl Flake Shareholder of: Myriad Genetics, Inc., Employee of: Myriad Genetics, Inc., Brent Mabey Shareholder of: Myriad Genetics, Inc., Employee of: Myriad Genetics, Inc. Jerry Lanchbury Shareholder of: Myriad Genetics, Inc., Employee of: Myriad Genetics, Inc.

DOI: 10.1136/annrheumdis-2021-eular.1657

\section{POS0457 CHANGES OF RF ISOTYPE PROFILE IN PATIENTS WITH RHEUSMATOID ARTHRITIS: DATA FROM 10 YEARS FOLLOW-UP STUDY}

M. R. Nur Aida Sabrina ${ }^{1}$, C. L. Too ${ }^{1,2}$, A. Haziqah Itqan $^{1}$, A. A. Siti-Aisyah ${ }^{1}$ A. F. Nurul-Aain ${ }^{1}$, L. K. Tan ${ }^{1}$, I. S. Lau ${ }^{3}$, M. Mohd Zain ${ }^{3}$, H. Baharuddin ${ }^{3}$, D. L. M. Ang ${ }^{4}$, A. R. Amnahliza ${ }^{4}$, P. S. $\mathrm{Ong}^{5}$, N. Mat Husin ${ }^{5}$, S. C. Gun ${ }^{6}$, N. Mohd Noor ${ }^{6}$, M. Z. Taib ${ }^{7}$, L. Padyukov ${ }^{2}$, L. Alfredsson ${ }^{8}$, L. Klareskog ${ }^{2}$ J. Rönnelid ${ }^{9}$, N. S. Shahril ${ }^{4} .{ }^{1}$ Allergy and Immunology Research Center, Institute for Medical Research, Ministry of Health Malaysia, Selangor, Malaysia; ${ }^{2}$ Department of Medicine, Division of Rheumatology, Karolinska Institutet and Karolinska University Hospital, Stockholm, Sweden; ${ }^{3}$ Department of Medicine, Selayang Hospital, Selangor, Malaysia; ${ }^{4}$ Department of Medicine, Putrajaya Hospital, Putrajaya, Malaysia; ${ }^{5}$ Department of Medicine, Raja Permaisuri Bainun Hospital, Perak, Malaysia; ${ }^{6}$ Department of Medicine, Tuanku Ja'afar Hospital, Negeri Sembilan, Malaysia; ${ }^{7}$ Medical Resource Research Centre, Institute for Medical Research, Ministry of Health Malaysia, Selangor, Malaysia; ${ }^{8}$ Institute of Environmental Medicine, Karolinska Institutet, Stockholm, Sweden; ${ }^{9}$ Department of Immunology, Genetics and Pathology, Uppsala University, Uppsala, Sweden

Background: Presence of autoantibodies such as anti-cyclic citrullinated peptide (anti-CCP2) and rheumatoid factor (RF) is of considerable diagnostic and prognostic value in patients with rheumatoid arthritis (RA). Limited data are available for autoantibody profile changes over time in patients with RA.

Objectives: Thus, we compared the presence of anti-CCP2 and different RF isotypes in individual RA patients at baseline and during 10 years follow-up. Methods: A total of 320 RA patients from the Malaysian Epidemiological Investigation of Rheumatoid Arthritis (MyEIRA) case-control study was included in this study. The presence of anti-CCP2, IgM RF, IgG RF, and IgA $\mathrm{RF}$ at baseline and at later time point $( \pm 10$ years) were determined using enzyme-linked immunosorbent assays, with identical techniques in paired samples. Seropositive RA is defined by the presence of at least one autoantibody, whilst seronegative RA is defined by the absence of all investigated autoantibodies.

Results: The proportion of seropositive RA were higher for the follow-up samples $(n=263,82.2 \%)$ as compared to the baseline samples $(n=251$ $78.4 \%$ ). Among the baseline samples, 105 (41.8\%) were positive for antiCCP2 and all RF isotypes. Of these individuals, 85 (81.0\%) remained pos itive for all antibodies at the follow-up, while $20(19.0 \%)$ lost one or more RF isotypes (4 IgM RF, 19 IgG RF and 13 IgA RF). Interestingly, 14 (5.6\%) RA patients who were seropositive at baseline became totally seronegative after follow-up. Among the 69 patients seronegative at baseline, 26 (37.7\%) acquired one or more autoantibodies at follow-up (14 IgM RF, 2 IgG RF, 9 IgA RF and 8 anti-CCP2) (Figure 1)

Conclusion: Anti-CCP2 present at baseline usually remained at follow-up Among Malaysian RA patients, changes in status were mainly found for RF of all isotypes.

REFERENCES:

[1] Barra, Lillian et al. "Lack of seroconversion of rheumatoid factor and anti-cyclic citrullinated peptide in patients with early inflammatory arthritis: a systematic literature review." Rheumatology (Oxford, England) vol. 50,2 (2011): 311-6.

[2] van Delft, Myrthe A M, and Tom W J Huizinga. "An overview of autoantibodies in rheumatoid arthritis." Journal of autoimmunity vol. 110 (2020): 102392. 

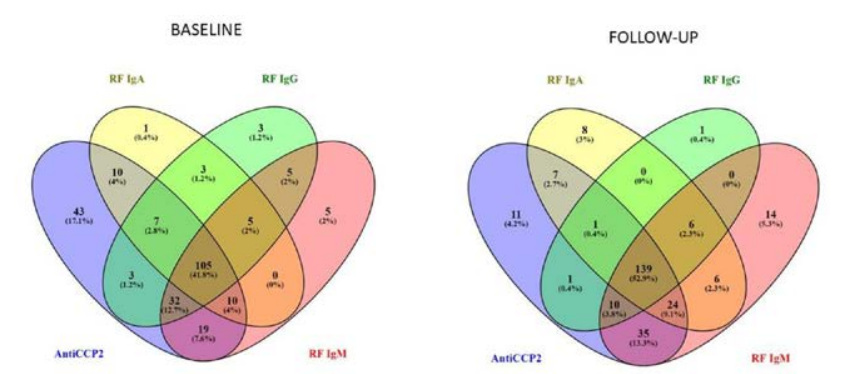

Figure 1. Comparison of serum autoantibody profile in rheumatoid arthritis patients during baseline enrolment and 10 years follow-up.

Acknowledgements: The authors would like to thank the Director General of Health, Ministry of Health Malaysia for supporting this study. The authors are also indebted to participants for their kind participation. This study was financially supported by the Ministry of Health, Malaysia (JPP-IMR 08-012; 18-051).

Disclosure of Interests: None declared

DOI: 10.1136/annrheumdis-2021-eular.1714

\section{POS0458 IDENTIFICATION OF HUB GENES AND MOLECULAR PATHWAYS IN PATIENTS WITH RHEUMATOID ARTHRITIS BY BIOINFORMATICS ANALYSIS}

L. Cheng ${ }^{1}$, S. X. Zhang ${ }^{2,3,4}$, S. Song ${ }^{2,3,4}$, C. Zheng ${ }^{1}$, X. Sun ${ }^{1}$, S. Feng ${ }^{1}$, T. Kong ${ }^{1}$, G. Shi ${ }^{5}$, X. Li ${ }^{2,3}$, P. F. He ${ }^{6}$, Q. Yu ${ }^{1,6} .{ }^{1}$ Shanxi Medical University, School of Management, Taiyuan, China; ${ }^{2}$ Shanxi Medical University, Department of Rheumatology, Taiyuan, China; ${ }^{3}$ Shanxi Li Xiaofeng Medical Groups, Department of Rheumatology, Taiyuan, China; ${ }^{4}$ Ministry of Education, Key laboratory of Cellular Physiology at Shanxi Medical University, Taiyuan, China; ${ }^{5}$ Shanxi Medical University, Basic Medical College, Taiyuan, China; ${ }^{6}$ Shanxi Medical University, Institute of Medical Data Sciences, Taiyuan, China

Background: Rheumatoid arthritis (RA) is a chronic, inflammatory synovitis based systemic disease of unknown etiology ${ }^{1}$. The genes and pathways in the inflamed synovium of RA patients are poorly understood.

Objectives: This study aims to identify differentially expressed genes (DEGs) associated with the progression of synovitis in RA using bioinformatics analysis and explore its pathogenesis ${ }^{2}$.

Methods: RA expression profile microarray data GSE89408 were acquired from the public gene chip database (GEO), including 152 synovial tissue samples from RA and 28 healthy synovial tissue samples. The DEGs of RA synovial tissues were screened by adopting the R software. The Gene Ontology (GO) and Kyoto Encyclopedia of Genes and Genomes (KEGG) pathway enrichment analysis were performed. Protein-protein interaction (PPI) networks were assembled with Cytoscape software.

Results: A total of 654 DEGs (268 up-regulated genes and 386 down-regulated genes) were obtained by the differential analysis. The GO enrichment results showed that the up-regulated genes were significantly enriched in the biological processes of myeloid leukocyte activation, cellular response to interferon-gamma and immune response-regulating signaling pathway, and the down-regulated genes were significantly enriched in the biological processes of extracellular matrix, retinoid metabolic process and regulation of lipid metabolic process. The KEGG annotation showed the up-regulated genes mainly participated in the staphylococcus aureus infection, chemokine signaling pathway, lysosome signaling pathway and the down-regulated genes mainly participated in the PPAR signaling pathway, AMPK signaling pathway, ECM-receptor interaction and so on. The 9 hub genes (PTPRC, TLR2, tyrobp, CTSS, CCL2, CCR5, B2M, fcgr1a and PPBP) were obtained based on the String database model by using the Cytoscape software and cytoHubba plugin ${ }^{3}$.

Conclusion: The findings identified the molecular mechanisms and the key hub genes of pathogenesis and progression of RA.

\section{REFERENCES:}

[1] Xiong Y, Mi BB, Liu MF, et al. Bioinformatics Analysis and Identification of Genes and Molecular Pathways Involved in Synovial Inflammation in Rheumatoid Arthritis. Med Sci Monit 2019;25:2246-56. doi: 10.12659/MSM.915451 [published Online First: 2019/03/28]

[2] Mun S, Lee J, Park A, et al. Proteomics Approach for the Discovery of Rheumatoid Arthritis Biomarkers Using Mass Spectrometry. Int J Mol Sci 2019;20(18) doi: 10.3390/ijms20184368 [published Online First: 2019/09/08]

[3] Zhu N, Hou J, Wu Y, et al. Identification of key genes in rheumatoid arthritis and osteoarthritis based on bioinformatics analysis. Medicine (Baltimore) 2018;97(22):e10997. doi: 10.1097/MD.0000000000010997 [published Online First: 2018/06/01]
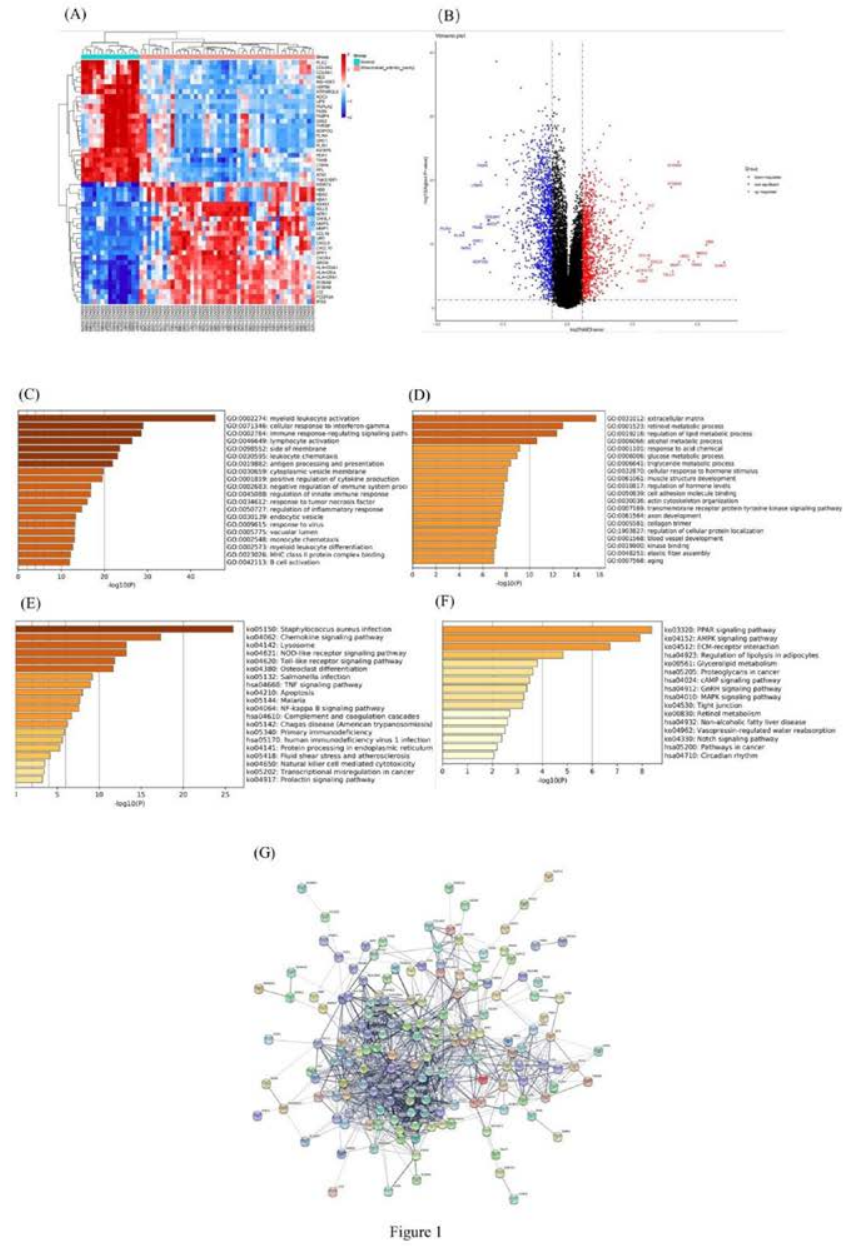

(A)Heat map of the top 50 up-regulated differentially expressed genes: Heatmaps of the differentially expressed genes (DEGs) including the 25 most upregulated and 25 most down-regulated genes. (B) Volcano map of gene expression: The horizontal axis is $\log 2 \mathrm{FC}$ and the vertical axis is $\log 10$ adj.p.val. Each dot represents a gene, blue represents down-regulated gene, red represents up-regulated gene, and black represents an in differentially expressed gene. (C,D)Visualization GO enrichment analysis: Go analysis showed that the function of up-regulated genes was mainly reflected in 20 aspects, and $\mathrm{B}$ cell activation had the lowest $\mathrm{p}$ value. The function of down regulated genes is mainly reflected in 20 aspects, and aging is the lowest in $P$ value. (E,F)KEGG pathway enrichment analysis: Among the signaling pathways of up-regulated genes, the largest P value was Staphylococcus aureus. PPAR signaling pathway has the highest $p$ value among the signaling pathways of down regulated genes. (G)Protein-protein interaction network of differentially expressed genes: The 9 hub genes were selected,
they were PTPRC, TLR2, tyrobp, CTSS, CCL2, CCR5, B2M, FCGR1A and PPBP.

Acknowledgements: This project was supported by National Science Foundation of China (82001740), Open Fund from the Key Laboratory of Cellular Physiology (Shanxi Medical University) (KLCP2019) and Innovation Plan for Postgraduate Education in Shanxi Province (2020BY078).

Disclosure of Interests: None declared

DOI: 10.1136/annrheumdis-2021-eular.1938

\section{POS0459 \\ UNSUPERVISED CLUSTERING IDENTIFIES UNIQUE SUBSETS OF PATIENTS IN A RACIALLY AND ETHNICALLY DIVERSE RHEUMATOID ARTHRITIS COHORT}

G. Luil ${ }^{1}$ E. Noss ${ }^{2}$, N. Singh ${ }^{2}$, J. Andrews ${ }^{2}$, J. Graf ${ }^{3}$, K. Wysham ${ }^{2,4} .{ }^{1}$ University of Washington, Bioengineering, Seattle, United States of America; ${ }^{2}$ University of Washington, Rheumatology/Medicine, Seattle, United States of America; ${ }^{3}$ University of California San Francisco Parnassus Campus, Rheumatology/ Medicine, San Francisco, United States of America; ${ }^{4}$ VA Puget Sound Health Care System, Rheumatology/Medicine, Seattle, United States of America

Background: Single biomarkers have limited utility to date in guiding RA clinical care. Machine learning algorithms may better identify and stratify RA patients with differential outcomes.

Objectives: To determine if unsupervised machine learning methods can be employed in a racially and ethnically diverse RA cohort to identify clusters of patients with different disease activity trajectories, as measured by DAS28ESR. Methods: Data are derived from the longitudinal, observational University of California, San Francisco RA Cohort. Along with routine labs, medications and disease activity assessments, a multiple biomarker of disease activity (MBDA) 